Journal of the Optical Society of Korea, Vol. 9, No. 2, June 2005, pp. 59-63

\title{
Three-beam Apodization in Photopolymer
}

\author{
Duc Dung Do and Nam Kim \\ Dept. of Computer \& Communication Eng., Chungbuk National University, KOREA \\ Kwon Yeon Lee \\ Dept. of Electronic Eng., Sunchon National University, KOREA \\ Seok Hee Jeon \\ Dept. of Electronic Eng., Incheon University, KOREA
}

(Received March 15, 2005 : revised April 14, 2005)

\begin{abstract}
In this paper, we present a novel apodization technique using three beams to fabricate an apodized grating in photopolymer. An orthogonal-polarization beam used to control the saturated modulation amplitude of the grating is added during recording time. As a result of the experiment, holographic apodized gratings with uniform, inverse Gaussian, and triangular profiles are fabricated successfully in DuPont's HRF-150-38 photopolymer.
\end{abstract}

OCIS codes : $090.7330,220.1230 \mathrm{vv}$

\section{INTRODUCTION}

The apodization technique and apodized gratings are well-known as the keys for suppressing the sidelobes and reducing the crosstalk in optical demultiplexer for the dense wavelength division multiplexing (DWDM) system [1, 2]. Another application utilizing apodized grating is a beam shaping, where the laser beam is reshaped into another form such as a flat-top distribution $[3,4]$, or conversely the uniform beam is turned back to Gaussian profile. In those fields, the fabrication of apodized grating intensively depends on the requirement of the optical system, i.e. when the size or apodizing profile is subject changed; all the recording system is set up again. On the other hand, the properties of holographic material are varied and nonlinear generally. Therefore, it is necessary to have a fabrication that is flexible and controllable to overcome those limits.

Over the past few years, photopolymers have reached a primary position in the variety of holographic recording materials [5]. They find applications in holographic display, optical data storage, optical demultiplexer, and holographic optical elements. To fabricate the apodized grating in photopolymer, the time evolution of index modulation in photopolymer, the dependence of the saturation diffraction efficiency on the recording intensity and on the beam intensity modulation must be known. In Ref. 6, Piazzolla and Jenkins presented a model that gave a simple closed-form expression with which one can predict a number of experimental observations. Basing on the model of the polymerization, we propose a method of fabricating apodized grating in photopolymer using an additional orthogonalpolarization beam, which is controlled by a spatial light modulator (SLM).

This paper is divided as follows. We first present the scheme of the three-beam recording method in section 2 . In section 3 , the verification of the photopolymer characteristic is analyzed. Finally, by the three-beam recording method, the trial of fabrication of several kinds of apodized gratings including uniform, inverse Gaussian, and triangular is described in detail.

\section{THREE-BEAM RECORDING SCHEME}

In recent years, there is much progress in manufacturing photopolymer. The photopolymer has several advantages over the other options. These advantages include high reliability, large thickness for volume application (up to $100 \mu \mathrm{m}$ ), high index modulation, dry processing, and low price. The final holograms recorded in photopolymer are insensitive to humidity and temperature and can be conveniently mounted onto paper, glass or plastic, or even embedded in acrylics. The ease of use and simple processing requirements allow these 
materials to be amenable to mass production of holographic optical elements.

A grating is built in the photopolymer due to the polymerization of monomers when they are exposed by coherent beams. At bright fringes of the interference pattern, the monomers are polymerized, which causes a decrease in the monomer concentration there. The concentration gradient between the dark and bright regions makes the monomers diffuse. After the exposure, the difference in the concentration of polymer in the material decides the index modulation of the grating, which in turn determines the diffraction efficiency of the grating as pointed out by H. Kogelnik [7]

$$
\eta=\sin ^{2}\left(\frac{\pi L \Delta n}{\lambda_{w} \cos \theta_{w}}\right)
$$

where $L$ is the thickness of the grating, $\lambda_{w}$ is the recording wavelength, and $\theta_{w}$ is the recording angle. Because the index modulation depends on the exposure time, the beam intensity, and the beam intensity modulation, we can use them to control the diffraction efficiency and to obtain the apodized grating. However, the response of the photopolymer to the exposure time and the recording beam intensity is non-linear and unstable. Meanwhile, the beam intensity modulation $\mathrm{m}$ is the only external factor that can affect and control the index modulation $\Delta n_{\text {sat }}$ at saturation ${ }^{6}$.

$$
\Delta n_{\text {sat }}=m \Delta n_{M}
$$

where $\Delta n_{M}$ is the maximum available index modulation. From Eq. (1) and (2), it is seen that the saturation diffraction efficiency of the grating intensively depends on the beam intensity modulation by

$$
\eta_{s a t}=\sin ^{2}\left(\frac{\pi L m \Delta n_{M}}{\lambda_{w} \cos \theta_{w}}\right) .
$$

Conclusively, if one can control the intensity modulation spatially, an apodized grating will be fabricated easily. In the case of the two recording beams, a varied index modulation can be obtained when the intensity of one beam is fixed and that of the other is spatially modified. However, a self-diffraction phenomenon that is a dynamical process of interaction between material and recording beams will happen. During the recording time, the growing grating couples the recording waves, changes their phase, amplitude, and the beam intensity modulation within the holographic region. The modified waves interfere and write an updated grating, which may be not uniform in amplitude, and may be not in phase with the preexisting one. Consequently, the self-diffraction in the photopolymer may cause the change of the grating geometry with non-uniform index modulation. Therefore, the phase and the intensity of the diffracted beam are unpredictable.

To overcome the self-diffraction problem, we propose an apodized grating-recording method using three beams in which one beam has a function to change the beam intensity modulation spatially. This third beam has the polarization mode perpendicular to those of the two others and therefore cannot make the interference with them. With that arrangement, the intensity pattern of the two interfering plane waves with the attendance of the third wave can be expressed as

$$
I(x)=I_{0}\left\lfloor 1+m \cos \left(K_{g} x\right)\right\rfloor
$$

where $I_{0}=I_{1}+I_{2}+I_{i}$ is the average recording intensity, $I_{1}, I_{2}$, and $I_{i}$ are intensity of the first, second and the third beams, respectively, $K_{g}=2 \pi / \Lambda_{g}$ is the grating wavenumber with the grating period $\Lambda_{g}$, and the beam intensity modulation is

$$
m=\frac{2 \sqrt{I_{1} \bar{I}_{2}}}{I_{0}}=\frac{2 \sqrt{I_{1} I_{2}}}{I_{1}+I_{2}+I_{i}}
$$

From here, if the intensities $I_{1}$ and $I_{2}$ are equal, Eq. (5) will become

$$
m=\frac{1}{1+\frac{B}{2}},
$$

where the beam intensity ratio is $B=\frac{I_{i}}{I_{1}}=\frac{I_{i}}{I_{2}}$.

Finally, from Eq. (3) and (6), the saturation diffraction efficiency will be controlled by the beam intensity ratio as follows

$$
\eta_{s a t}=\sin ^{2}\left(\frac{\beta}{2+B}\right)
$$

where $\beta=\frac{2 \pi L \Delta n_{M}}{\lambda_{w} \cos \theta_{w}}$ is a constant describing the recording condition and the material. Now, a desired apodized grating can be obtained if one modulates the third beam intensity by such a function that

$$
B(x, y) \propto \frac{1}{\arcsin (\sqrt{\eta(x, y)})} .
$$

In order to do that, a Spatial Light Modulator (SLM), which is capable of converting data in electronic form into spatially modulated optical signals, is a useful device. It is remarkable that the third beam modulated by the SLM just controls the index modulation but does not take part in the energy-exchanging process. As a result, the self-diffraction phenomenon does not occur. Moreover, by use of the SLM, gratings with various sizes and profiles are fabricated easily and 
flexibly. Basing on this scheme, we have fabricated several gratings with different profiles in HRF-150-38 photopolymer. The results will be presented in following sections.

\section{PHOTOPOLYMER CHARACTERISTICS}

Firstly, before applying the scheme of the threebeam recording, the saturation diffraction efficiency characteristic of the material when the beam intensity modulation is varied should be verified. A number of transmission holograms in the HRF-150-38 photopolymer are exposed and monitored in the experimental setup that is shown in Fig. 1. The laser beam from a continuous wave (CW) Nd-YAG source (wavelength $\lambda_{w}=532 \mathrm{~nm}$ ) is filtered by a spatial filter, collimated by a lens, and passed centrally by an aperture to ensure that its intensity has a uniform distribution. The recording beams normally polarized to the plane of incidence are symmetrically interfering, each incident with an angle $\theta_{w}$ of $15^{\circ}$ in air with respect to the holographic plate normal. The half wave plates $\mathrm{H} 1$ and $\mathrm{H} 2$ are used to change the intensity ratio. The shutter controlled by a computer blocks a beam for every 10 s. The detectors collect the intensity data of the diffracted and transmitted beams at the blocking time. The diffraction efficiency is calculated by the ratio of the intensity of the diffracted beam to the total output intensity.

Relied on this experimental setup, a thorough investigation has been performed. The average intensity is fixed at $0.4 \mathrm{~mW} / \mathrm{cm}^{2}$, and the intensity modulation is varied according to values. $1,0.95,0.85,0.8,0.6,0.45$, $0.35,0.25,0.17$, and 0.1 . The results that present the growth of the diffraction efficiency versus the exposure time are shown in Fig. 2. In all cases, the diffraction efficiencies grow up after the photopolymer is exposed for 250s. The saturation diffraction efficiency highly

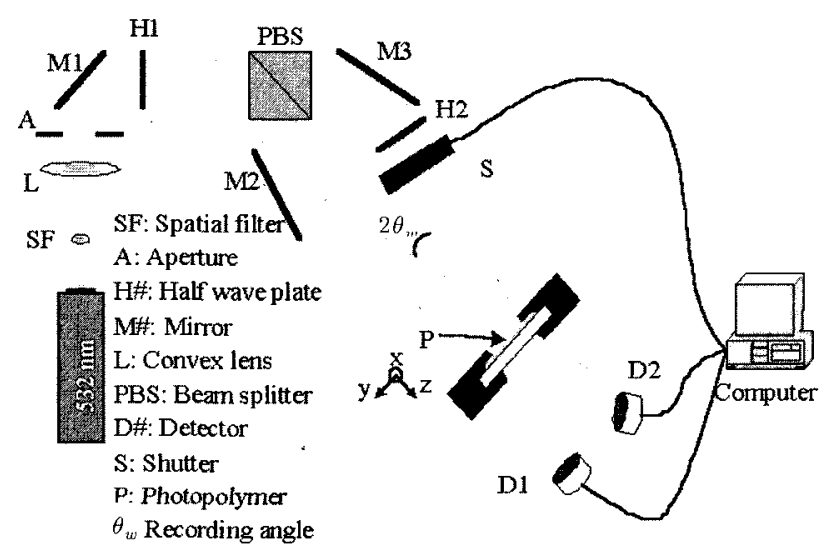

FIG. 1. Experimental setup to investigate the characteristic of the photopolymer.

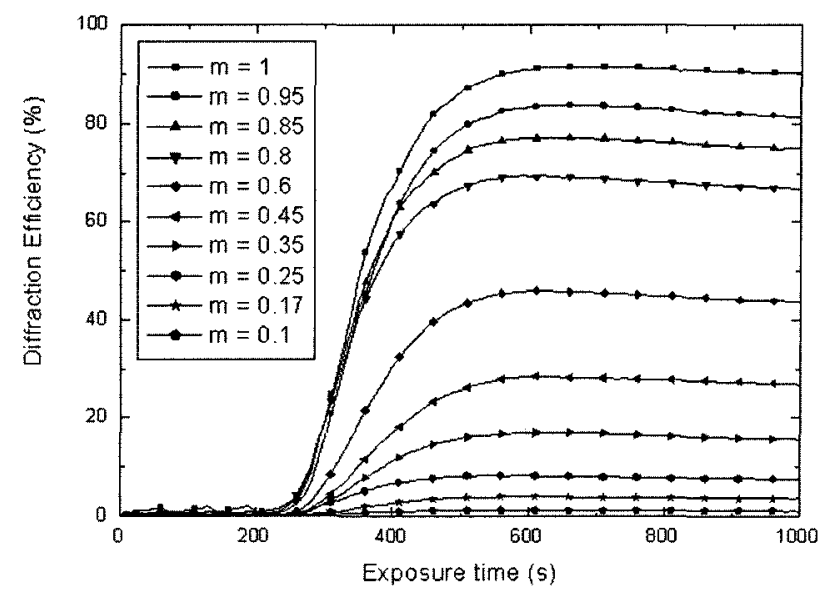

FIG. 2. Diffraction efficiency of the photopolymer depends on the exposure time.

depends on the beam intensity modulation $\mathrm{m}$ as shown in this figure.

FIG. 3. shows the saturation diffraction efficiency versus the beam intensity modulation. The solid line is a predicted saturation diffraction efficiency according to the value $\beta$ calculated from measured value for $m=1$. It is obvious that the saturation diffraction efficiency exactly follows the rule described in Eq. (3). This result once again confirms that we can determine the pattern, which is put into the SLM during the recording time to obtain the desired diffraction efficiency profile of apodized grating.

\section{APODIZED GRATING FABRICATION}

Based on three-beam recording scheme in the photopolymer as mention above, a fabrication of several apodized gratings is implemented. In our experiment, the uniform, inverse Gaussian, and triangular profiles

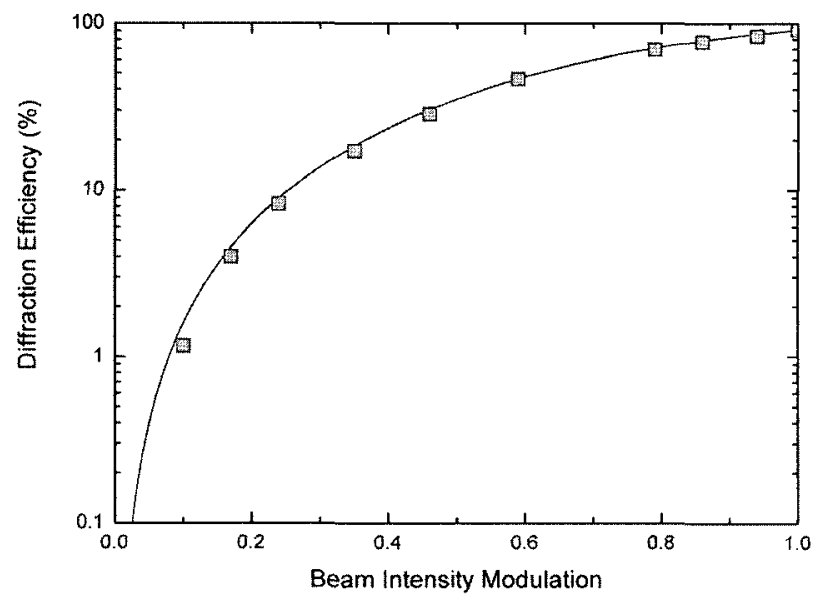

FIG. 3. Saturation diffraction efficiency of the photopolymer depends on the beam intensity modulation. 
are used for demonstration. Fig 4 shows the scheme of the recording system. The laser light of 532-nm wavelength emitted from the Nd:YAG laser source is spatially filtered and collimated by lenses. Intensity of each two beams in s-polarization mode is fixed at 0.2 $\mathrm{mW} / \mathrm{cm}^{2}$. The third beam has p-polarization mode to ensure that it does not make interference with two others. The intensity of the additional beam is modulated by a $640 \times 480$-resolution liquid crystal display (LCD). The data put into the LCD should have the form as being pointed out in Eq. (8). Besides, a spatial filter, which includes two lenses and an aperture, is used to block all the high diffraction orders created by the LCD. The recording material is placed in the interference region of two beams in around $600 \mathrm{~s}$. As long as this time, all monomer is polymerized, and the diffraction efficiency is saturated.

To measure the profile of recorded gratings, the 633-nm He-Ne laser beam is used as a probe. After being created, the grating is horizontally shifted over the probe beam by a linear stage as depicted in Fig. 5. A computer automatically collected the intensity value of the diffracted light, which is measured by the detector. A note is that the probe beam should have

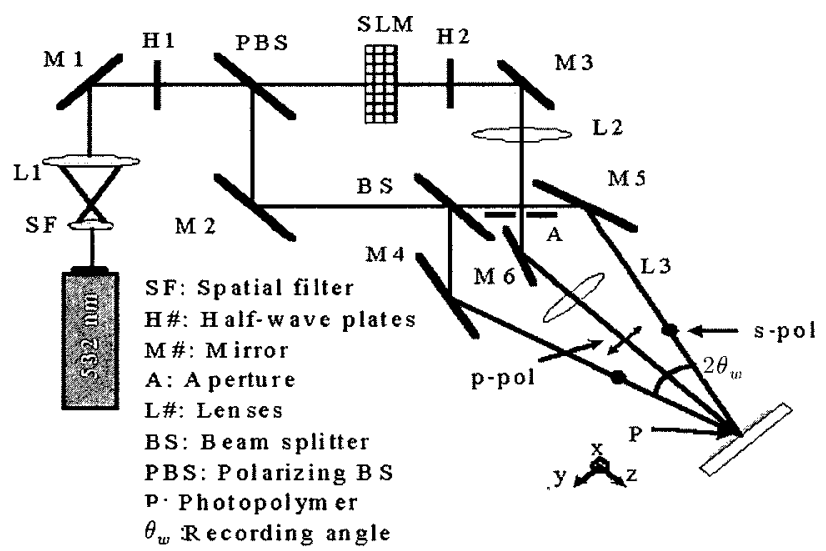

FIG. 4. Experimental setup to record the apodized grating.

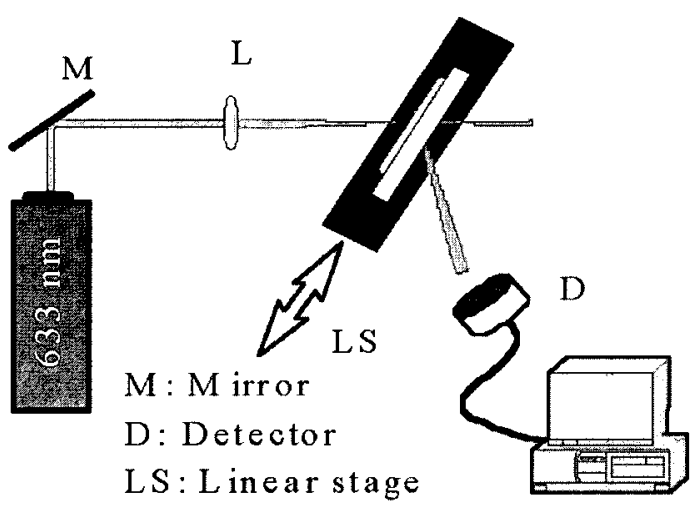

FIG. 5. Experimental setup to measure the grating profile.

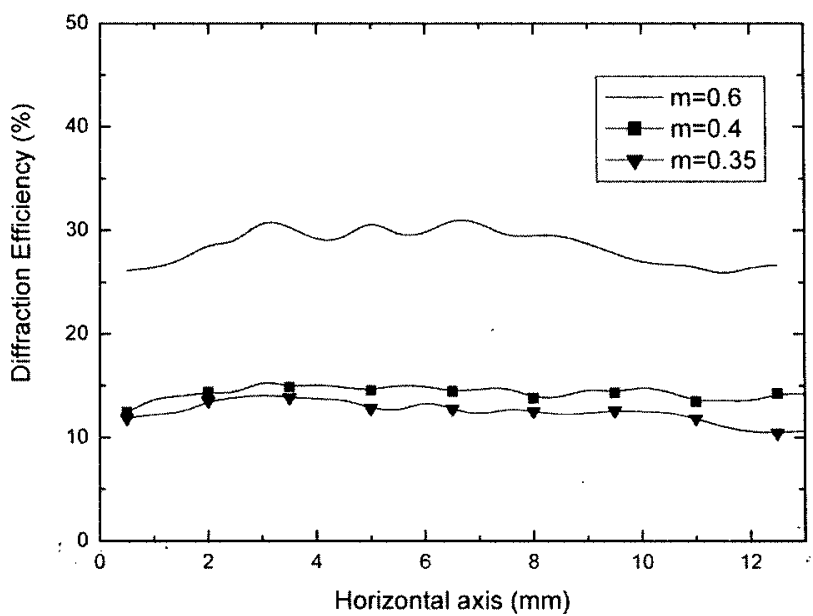

(a)

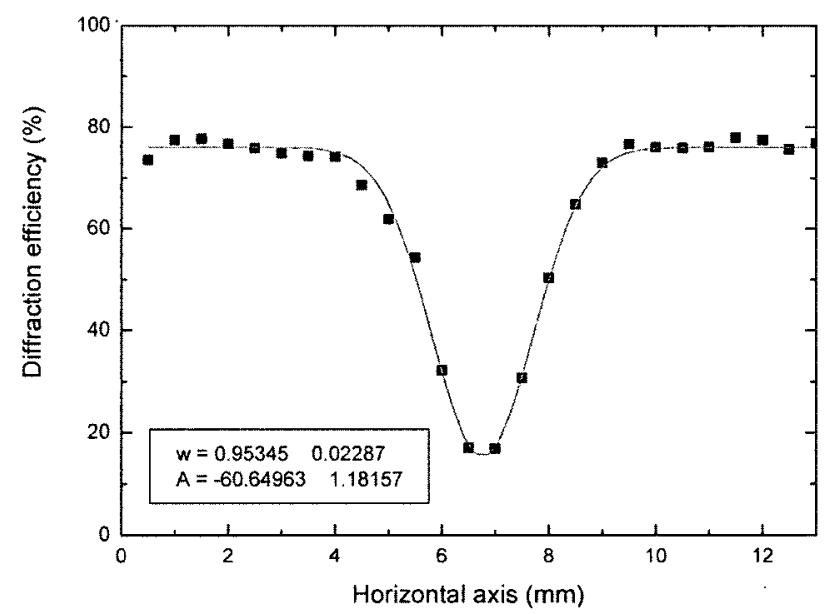

(b)

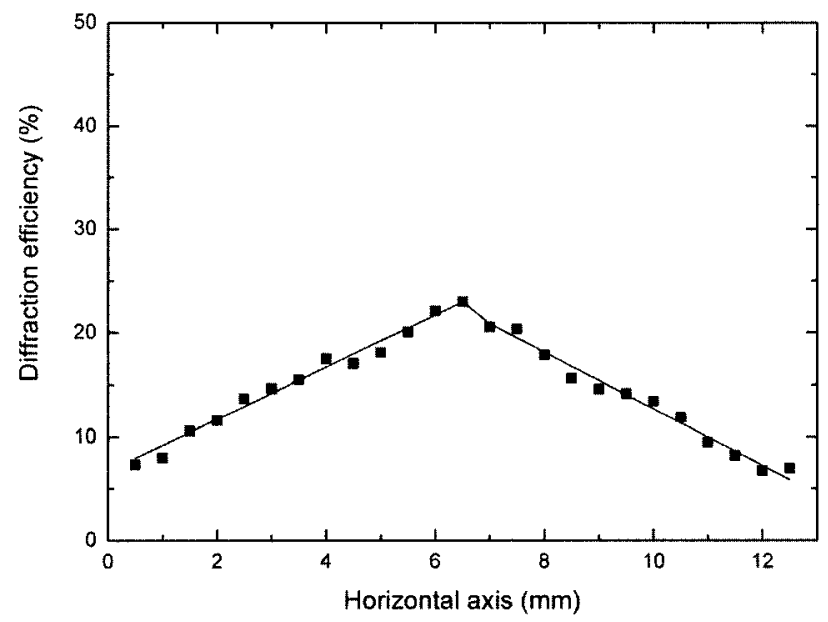

(c)

FIG. 6. Gratings recorded by orthogonally-polarized beam with a) uniform, b) inverse Gaussian, and c) triangular profiles.

a small spot size to distinguish any fast variation of the modulation amplitude along the apodized direction. In our experiment, a focused Gaussian beam with $10-\mu$ 
$m$ spot size is employed.

Fig. 6 shows the results of gratings of 13-mm width created and measured by the above fabricating process. In the case of the uniform grating, the beam intensity modulations are chosen equal to $0.6,0.4$, and 0.35 , at which the predicted diffraction efficiencies are $45 \%$, $23 \%$, and $17 \%$, respectively. However, as results of the experiment, the according values are $30 \%, 15 \%$, and $12 \%$, as shown in Fig. 6(a). It is understood that the diffusion of monomers is strongly suppressed when photopolymer is exposed under the high average intensity because the tacky composition gels and hardens quickly in this condition. For this reason, the diffraction efficiencies are smaller than expected.

For the case of the Gaussian grating, a Gaussian function is used to fit the measured data. The fitting line has the standard deviation $\mathrm{w}$ of about $1 \mathrm{~mm}$ and the amplitude $A$ of $60 \%$, as shown in Fig. 6(b). The maximum and minimum intensity modulations is fixed at 0.85 and 0.4 resulting in the highest and lowest diffraction efficiencies of $76 \%$ and $16 \%$, respectively. Those values in case of the triangular grating are 0.45 and 0.3 , and the respected diffraction efficiencies are $24 \%$ and $8 \%$, respectively, as shown in Fig. 6(c). The lower diffraction efficiencies than predicted values in all cases have the same reason as mentioned above. From the figure, it is clear that the measured profiles are not exactly matched to the ideal cases. This is due to the fringes happening inside the SLM. To avoid this problem, another spatial light modulator that has good optical quality such as deformable micro-mirror device (DMD) should be used.

\section{CONCLUSION}

In this paper, we have considered the apodizedgrating-recording method using an additional beam to control the diffraction efficiency. This three-beam method helps avoiding the unwanted self-diffraction phenomenon inherent to the photopolymer. Moreover, the diffraction efficiency of the apodized grating is controlled exactly as desired because the non-linear response of the photopolymer to the low recording beam is overcome. By utilizing LCD, we have demonstrated that it is possible to create gratings in various sizes and profiles without changing recording setup. In the experiment, the apodized gratings with three kinds of profiles, which are uniform, inverse Gaussian, and triangular, are successfully recorded. In conclusion, we have seen that the three-beam apodization technique is useful and applicable to fabricate holographic elements for applications such as laser diode beam shaping and low cross-talk filtering.

\section{ACKNOWLEDGEMENT}

This work was supported by grant No.(2001-0437) from University of Incheon

* Corresponding author : ddd@osp. chungbuk. ac.kr

\section{REFERENCES}

[1] D. D. Do, S. K. Park, J. W. An, N. Kim, and K. Y. Lee, "Gaussian apodized volume grating to suppress the crosstalk in holographic demultiplexers using highefficiency photopolymers," Opt. Eng., 43, 2022-2027, Sept. 2004.

[2] D. Lande, S. S. Orlov, and L. Hesselink, "Two-photon apodization in lithium niobate," Opt. Lett., 23, 3991401, Sept. 1998.

[3] M. Miler, I. Aubrecht, and J. Pala, "Holographic Gaussian to flat-top beam shaping," Opt. Eng., 42, 3114-3122, Nov. 2003.

[4] C. C. Aleksoff, K. K. Ellis, and B. D. Neagle, "Holographic conversion of a Gaussian beam to a near-field uniform beam," Opt. Eng., 30, 537-543, May 1991.

[5] B. L. Booth, "Photopolymer material for holography," Appl. Opt., 14, 593-601, 1975.

[6] S. Piazzolla and B. K. Jenkins, "Holographic grating formation in photopolymers," Opt. Lett., 21, 1075-1077, June. 1996.

[7] H. Kogelnik, "Coupled wave theory for thick hologram gratings," Bell Tech. J., 48, 2909-2947, Nov. 1969. 